\title{
Un nuevo dato sobre la enajenación de bienes de la Corona: la venta de las Alcabalas de Yegros (Toledo)
}

\author{
Paulina López Pita *
}

Situada en las proximidades de la villa de Mora (Toledo), se encuentra la dehesa de Yegros la cual desde comienzos del siglo XIII, momento en el que encontramos por vez primera datos específicos sobre ella en las relaciones documentales, pertenecía a la Orden de Santiago.

Sabemos que en 1150 Yegros fue donada por el rey Alfonso VII al Concejo de San Vicente pero, pocos años más tarde, en 1219 concretamente cuando Fernando III entregó a la Orden de Santiago el Hospital de Toledo, le hizo entrega asimismo de la heredad de Yegros, la cual junto con el Hospital de los caballeros de la ciudad de Toledo, el de Santiago de Dos Barrios, y diversas heredades en el alfoz de Ávila, Dos Barrios, Maqueda, Mascaraque, ciertos lugares de Toledo y dos dehesas (la Fuente del Majuelo, en el Campo de Montiel y la Alameda, en León) formaban parte de la Encomienda del Hospital de Toledo'.

Fue a mediados del siglo XVI, época en la cual la Hacienda Regia se encontraba agobiada por las grandes necesidades económicas a las que debía hacer frente, cuando el rey Felipe II acordó vender las alcabalas de la dehesa de Yegros que le pertenecían como administrador que era

* UNED.

- Cfr. P. A. Porras Arboleda: Los señoríos de la Orden de Santiago en su provincia de Castilla durante el siglo xv, Tesis Doctoral. Universidad Complutense, 1982, t.ll, pp. 471 y siguientes. 
de las Ordenes Militares. Era ésta una medida que el monarca se vio obligado a tomar en varias ocasiones debido a los cuantiosos gastos que debía afrontar la Corona ocasionados en buena parte por su propia grandeza que le había llevado a ocuparse de asuntos bastante distintos a la vez que dispersos. $Y$, a pesar de que el rey conocía el daño y el perjuicio que esta medida ocasionaba, no se pudo evitar que se continuasen vendiendo bienes pertenecientes a la Corona. De tal forma que a finales del siglo XVI la Junta formada por Felipe II para consultarle nuevos arbitrios, no encontraba otros, sino la venta de vasallos y jurisdicciones, alcabalas, tercias y otras rentas perpetuas.

En tales circunstancias el rey Felipe II haciendo uso de la facultad que tenía para desmembrar bienes de las Ordenes Militares y encomiendas y hacer uso de ellas en cantidad de 40.000 ducados, según diversas bulas, breves y letras apostólicas que le habian sido concedidas por los Pontífices ${ }^{2}$, acordó, en junio de 1569, con Francisco de Rojas, regidor de la ciudad de Toledo, la venta de la villa de Mora, que con anterioridad había enajenado de la encomienda de la Orden de Santiago ${ }^{3}$, junto con las alcabalas de la villa de Layos, perteneciente al regidor y sobre la cual se había instituido mayorazgo ${ }^{4}$, y la venta asimismo de las alcabalas de la dehesa de Yegros que ahora nos ocupa:

«Lo que por mandado de su magestad se asienta y conçierta con don Francisco de Rojas, vezino y regidor de la ciudad de Toledo ... que se aya de hazer desde luego averiguaçión de las Alcavalas a su magestad perteneçientes en la dehesa de Yegros que es del Hospital de Santiago de la çiudad de Toledo para que su magestad venda al dicho don Francisco, las alcavalas de la dicha dehesa y su término, cargándole por ellas lo que fuere segun la dicha averiguaçión que dello se hiciese a razon de los dichos treynta y ocho mil maravedís... "s .

Cuando esta venta se lleva a cabo, ya mediado el siglo XVI, hacía tiempo que Yegros estaba despoblado. No obstante hay numerosos tes-

${ }^{2}$ Bula del papa Clemente VII de 20 de septiembre de 1529 , confirmada por el papa Paulo III el 17 de agosto de 1536, confirmada a su vez por el papa Pío IV, el 21 de noviembre de 1569.

${ }^{3}$ P. López PITA: Layos, origen y desarrollo de un señorío nobiliario: el de los Rojas, Condes de Mora, Toledo, Caja de Ahorro, 1988, pp. 129 y sigs.

4 P. Lopez Pita: Op. cit., p. 228.

${ }^{5}$ Archivo Conde de Mora, Leg. 1, n. 2. 
timonios que nos hablan de que en otro tiempo debió residir en ella una población estable e importante numéricamente, a juzgar por la serie de edificios que, aunque derruidos, atestiguan que en ellos hubo un hábitat de relativa importancia ${ }^{6}$, sin embargo en 1569 , cuando se averigua el valor de la dehesa, únicamente quedaba en pie una casa, que pertenecía a la Orden de Santiago, "con su carcel, horca y picota», donde se juzgaban los delitos que se hubiesen cometido dentro del término de Yegros $^{7}$.

Yegros era, según la propia declaración que en el citado año hicieron los ganaderos que pastaban en su término ${ }^{8}$, dehesa cerrada, como consta por la mojonera que señala los términos con los que confina. Su extensión, de modo aproximado, era de legua y media de largo, desde el mojón que marca el límite de la villa de Mora hasta el mojón que señala el comienzo del término de la villa de Huerta de Valdecarabanos; y de una legua, poco más o menos, desde el término de Camporey hasta el término del Castillo que pertenece a Mora (Véase mapa adjunto) ${ }^{9}$.

Todo el espacio que integraba la dehesa se destinaba fundamentalmente para tareas agrícolas y ganaderas, constituyendo ambas los aprovechamientos básicos de la misma, distribuidos de la siguiente forma:

- Unas 2.000 fanegas, poco más o menos, estaban destinadas para tierra de labor, las cuales producían, aproximadamente, unas 7.000 fanegas de pan por mitad, trigo y cebada, y eran labradas en su mayor parte por los vecinos de Mora y de Mascaraque quienes pagaban por su arrendamiento al Hospital de Santiago 10

6 P. A. Porras Arboleda. Op. cit., p. 473.

7 A.G.S. Hacienda 300.

${ }^{8}$ Francisco de Segovia, Anton de Urda, Miguel Sánchez de Segovia, Pedro Valero, Francisco Maestro, Diego Martínez, Francisco Hernández de Segovia, vecinos de Mora; y Francisco de Mora vecino de Huerta de Valdecarabanos, Francisco Martín Aguado y su hermano Pedro de Mora, vecinos de Yepes, Bartolomé Martínez, Diego García Romero y Juan Díaz de Toro.

${ }^{9}$ La relación concreta de los mojones que señalaban los límites de la dehesa de Yegros eran los siguientes: mojón de Camporey, majuelo del Bachiller Juan Maesso (clérigo), majuelo de Diego Martín (escribano), camino de Arricoque, Huerta de Francisco Fernández de Segovia, camino principal de Mora a Yegros, haza de Juan Gómez, camino del carril, camino de la cañada, falda de la sierra que llaman "Cabeza del pendón», mojón en la cumbre de la sierra, valdíos que liaman de "navajuelo", cañada vieja, cañada del Horniilo, hoya de la butrera, camino de Tembleque a Toledo, río Algodor y mojón al cabo del charco del Castillo. A.G.S. Hacienda 300. 
ducados por yugada de tierra de labor (50 fanegas de sembradura).

- Unas 1.700 aranzadas de viñas y majuelos que se repartían entre los vecinos de Mora y Mascaraque, los cuales poseían 1.000 y 600 respectivamente, el resto se repartía entre diversos vecinos de la ciudad de Toledo que tenían heredades en este término. Cada uno de ellos pagaba al citado Hospital un real por aranzada.

En su mayor parte las viñas eran de reciente plantación, pues habían sido plantadas 6 ó 7 años antes de llevarse a cabo la dicha averiguación, por lo que solamente desde 2 ó 3 años atrás habían comenzado a dar su fruto experimentando desde su comienzo un notable incremento, así por ejemplo en 1566 se obtuvieron unas 1.000 arrobas de vino, al año siguiente unas 2.000, alcanzándose en 1568 las 3.000 arrobas. Algo similar ocurría con los 5.000 olivos que se habían plantado entre las viñas, que por ser muy nuevos, comenzaban por aquellos años a dar fruto.

- El resto de la dehesa estaba destinada para pasto de ganado, especialmente para ganado lanar y cabrío, ovejas, corderos, cabras, carneros, y machorras, con claro predominio del ganado lanar. $Y$ se hallaba dividida para su arrendamiento en 4 cuatros, que solían ajustarse por un período de 9 años, que cumplía el día de San Miguel, con vecinos de Mora, y de Huerta de Valdecarabanos especialmente. 
Un nuevo dato sobre la enajenación de bienes de la Corona

\begin{tabular}{|c|c|c|}
\hline CUARTOS & $\begin{array}{c}\text { PRECIO } \\
\text { ARRENDAMIENTO ANUAL } \\
\text { (Maravedís) }\end{array}$ & $\begin{array}{c}\text { N. }{ }^{\circ} \text { DE CABEZAS QUE } \\
\text { PASTABAN } \\
\text { (Invernadero-Agostadero) }\end{array}$ \\
\hline $\begin{array}{l}\text { LOMO DEL } \\
\text { ARROYO } \\
\text { Y PEDRERA }\end{array}$ & 194.000 & 1.800 \\
\hline $\begin{array}{c}\text { LA HIGUERUELA } \\
\underset{Y}{Y} \\
\text { HORNILLO }\end{array}$ & 170.000 & 1.200 \\
\hline $\begin{array}{c}\text { LAS PARRILLAS } \\
\text { BAJAS }\end{array}$ & 150.000 & 1.500 \\
\hline $\begin{array}{l}\text { LAS PARRILLAS } \\
\text { ALTAS }\end{array}$ & 167.000 & 1.400 \\
\hline
\end{tabular}

- Una pequeña parte se utilizaba para el cultivo de diversos productos de huerta: verduras, frutas, hortalizas, berenjenas, cebollinos, nabos, etc... cuya producción era muy baja, e incluso, hubo algún año, en que todo lo plantado se perdió.

Conocido esto y en cumplimiento de lo acordado entre Felipe II y Francisco de Rojas, el rey dio órdenes al juez Fermin Cruzat para que llevase a cabo la averiguación del valor de las alcabalas de la dehesa de Yegros durante los cinco años que transcurrieron entre 1564 y 1568, tanto de los ganados y frutos que en la citada dehesa se cogían como de los mantenimientos y mercaderías que se hubiesen vendido, calculándose asimismo su valor si de todo ello se llevase el 10 por 100 .

Por tal motivo le fue presentada al monarca una relación a todo ello tocante por parte del mayordomo del Hospital de Santiago de la ciudad de Toledo, como persona que tenía a su cargo los bienes y rentas del dicho Hospital, con quien estaba encabezada la dehesa; y por Hernando de la Losa, administrador del mismo. A través de la cual hemos podido conocer que: 
- Las alcabalas de Yegros estaban encabezadas con el Hospital de Santiago, por 13 años, que habían comenzado en 1564, por precio de 6.000 maravedís en cada uno de ellos.

- Durante los años $1564,1565,1566$ y 1567 las alcabalas habían sido cobradas y administradas por el dicho Hospital y de ello se habían ocupado personas a él pertenecientes, los cuales habían cobrado, durante ese tiempo, según consta en las partidas de los libros que presentaron, 650 maravedís, al llevar a razón de 20 mrs. el millar del total de los 32.471 mrs. que habían cobrado. (Véase apéndice I).

Asimismo y en esos cuatro años, llevaron para el Hospital 27.675 maravedís, que le correspondian del $1.383 .773 \mathrm{mrs}$. precio total de las ventas de viñas y majuelos que se habían efectuado durante el tiempo de la averiguación, (al cobrarse por alcabala a razón de 20 el millar). (Véase apéndice II).

- En 1568 el Hospital arrendó las alcabalas de Yegros a Juan de Palencia, vecino de la villa de Mora, por valor de 12.000 maravedís, igual cantidad que estaba pagando en 1569 cuando se lleva a cabo esta averiguación. Sin embargo no ha sido posible conocer cual fue el valor de las alcabalas de tierras y heredades que se vendieron en la dehesa, término y jurisdicción durante el año de 1568 , por no haberse elaborado ningún libro de cuentas por parte del arrendador.

Unicamente hemos podido saber, a través de los datos que se han conservado, que al igual que el Hospital, Juan de Palencia llevó 20 maravedís por millar, y que había cobrado $750 \mathrm{mrs}$. de alcabala de ciertos nabares y melonares que se vendieron en Yegros. $Y$ que asimismo tenía concertado con los arrendadores de los distintos cuartos destinados para el pasto del ganado, el cobro de 8 reales en cada año por el alcabala de lo que vendiesen en la dehesa, cuyo valor, por término medio era el siguiente: 


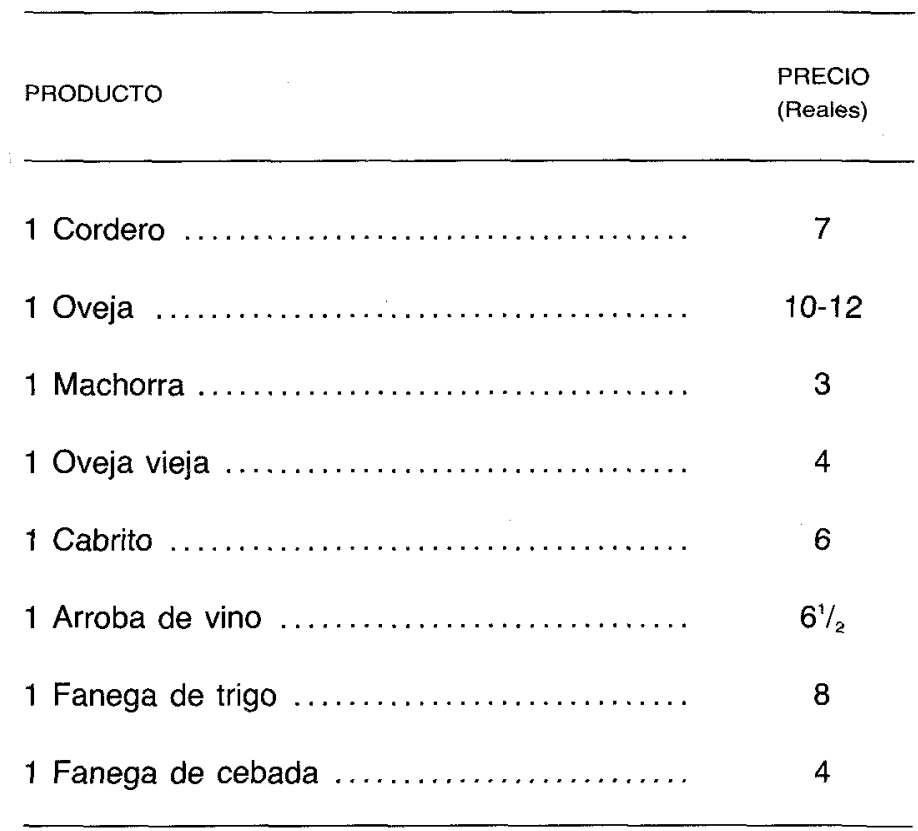

A través de lo expuesto hemos podido apreciar que al ser Yegros una dehesa despoblada en el siglo XVI tenía una excasa actividad comercial. Los herbajeros traían sus mantenimientos de otras partes, lo mismo que hacian las personas que acudian a la labranza y labores de las viñas, provenientes en su mayor parte, como dijimos, de Mora, Mascaraque y Toledo.

Por lo cual era realmente muy poco lo que se vendía en el término de Yegros, ya que la venta de la lana y corderos la efectuaban en la villa de Mora, donde conducían a sus ganados para esquilar, y en Orgaz principalmente, aunque en ocasiones ciertos productos de huerta se hubieron vendido en Tembleque.

Vemos pues que desde el punto de vista económico la venta de las alcabalas de la dehesa de Yegros no suponía un beneficio demasiado importante para el beneficiario, Francisco de Rojas, sin embargo, con ella el rey trataba de recompensar a quien tantos trabajos le había prestado. 


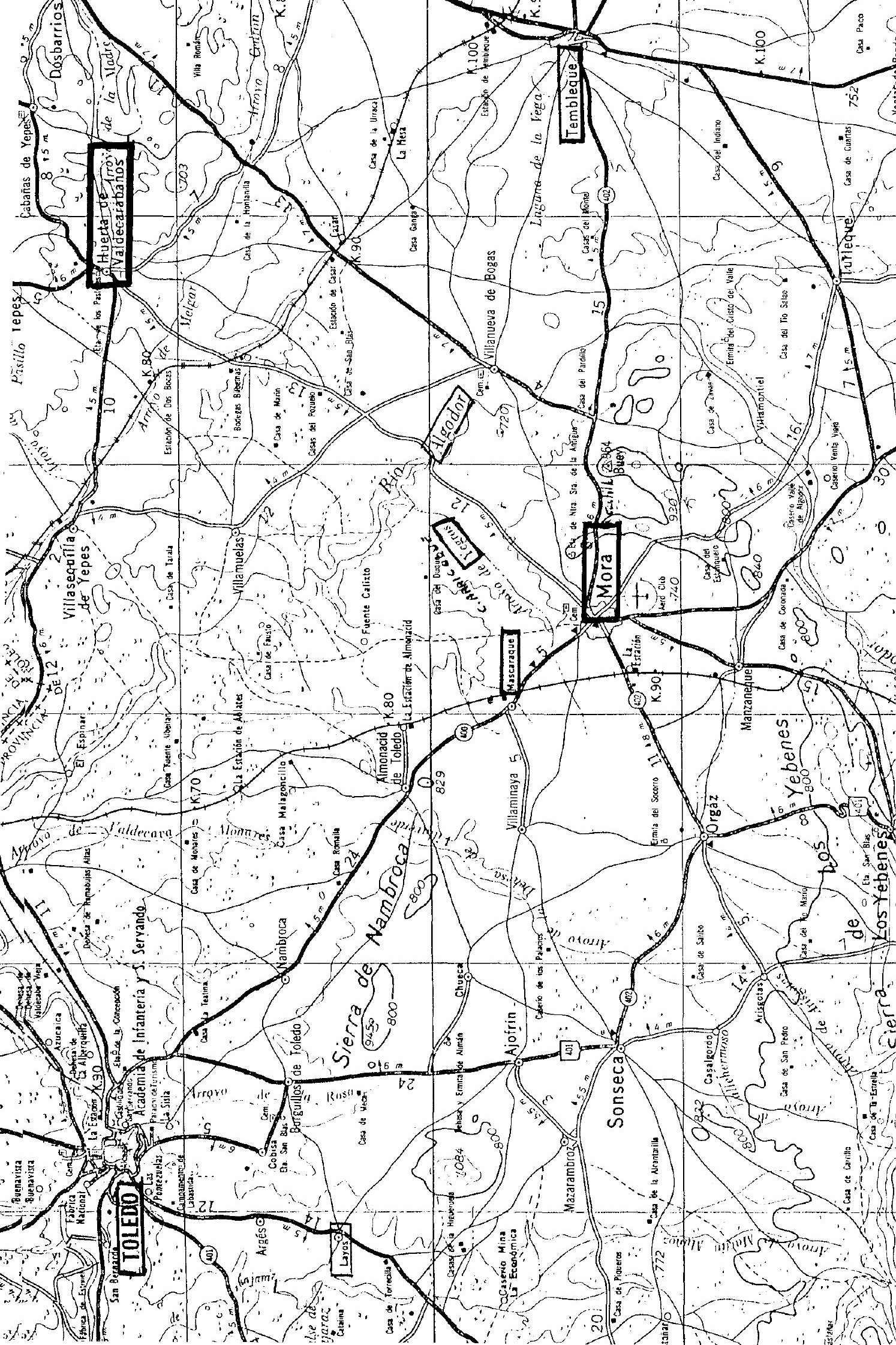




\section{APÉNDICE I}

RELACIÓN DE LO QUE SE COBRÓ DE ALCABALAS EN LOS AÑOS 1564 A 1567

Mrs.

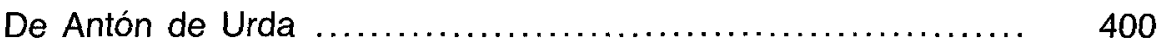

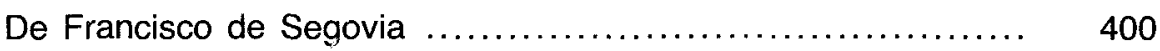

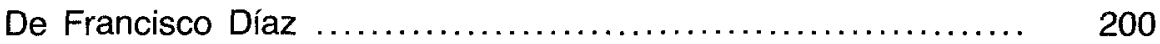

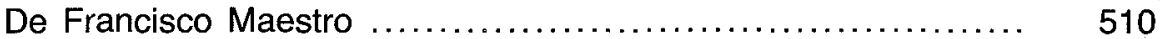

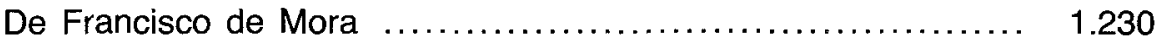

De Cristóbal Gómez .................................... 102

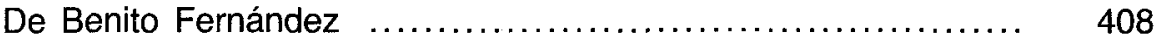

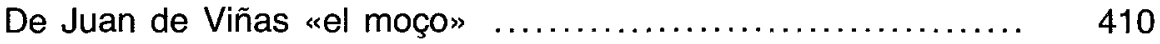

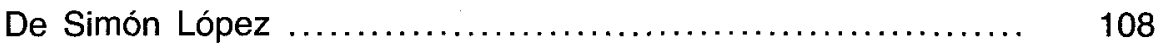

De L. López ........................................ $\quad 110$

De los hijos de Gabril Miñez $\ldots \ldots \ldots \ldots \ldots \ldots \ldots \ldots \ldots \ldots \ldots . . \ldots \ldots$

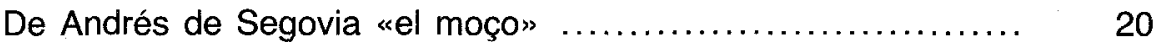


Mrs.

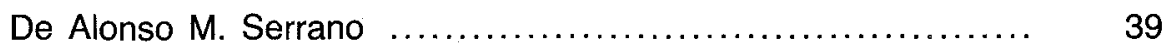

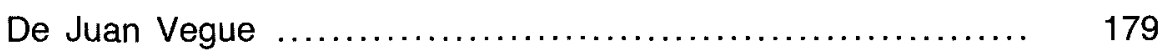

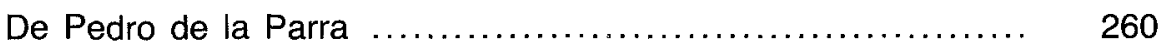

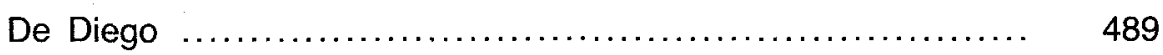

De Alonso López de Alameda $\ldots \ldots \ldots \ldots \ldots \ldots \ldots \ldots \ldots \ldots \ldots \ldots$

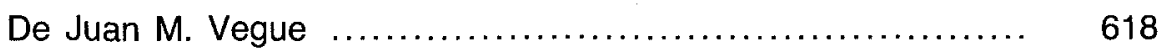

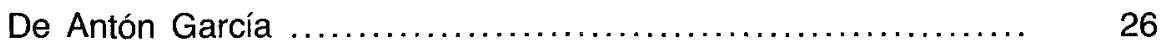

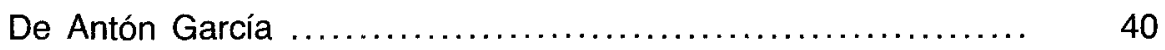

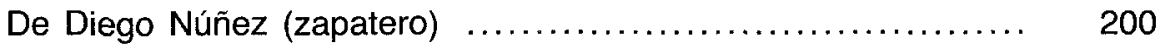

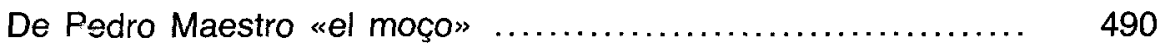

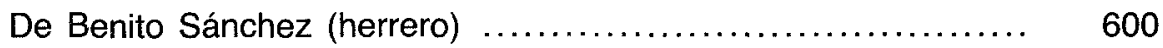

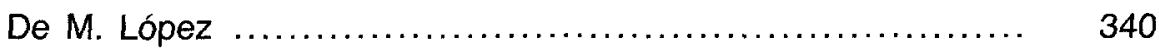

De Pedro Ruiz y Juan Sánchez ......................... 310

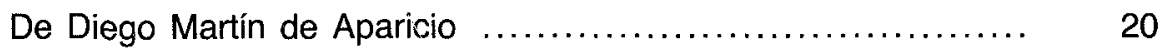

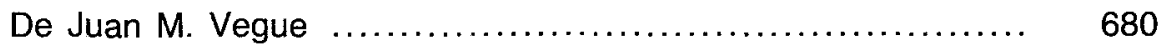

De Cristóbal García (tendero) $\ldots \ldots \ldots \ldots \ldots \ldots \ldots \ldots \ldots \ldots \ldots \ldots \ldots \ldots \ldots \ldots \ldots \ldots \ldots \ldots$

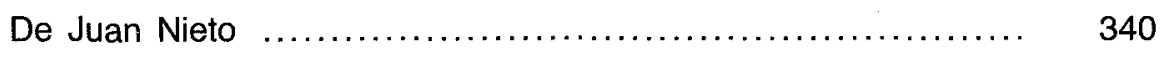

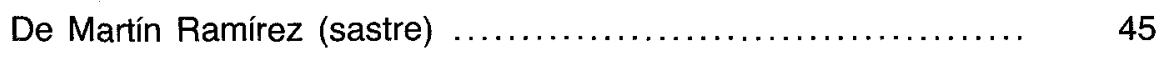

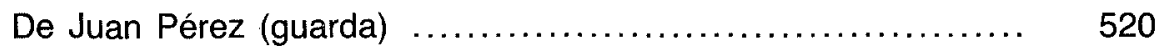


Mrs.

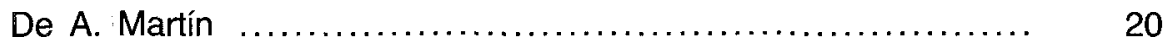

VECINOS DE MASCARAQUE

De Juan Delgado $\ldots \ldots \ldots \ldots \ldots \ldots \ldots \ldots \ldots \ldots \ldots \ldots \ldots \ldots \ldots \ldots \ldots \ldots \ldots, \quad 300$

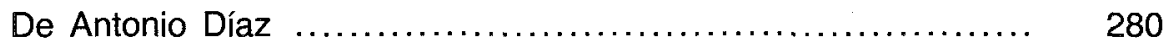

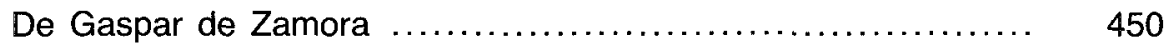

De Gonzalo S. ...................................... $\quad 480$

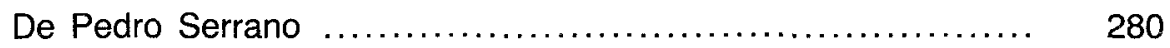

De Pedro de Galvez «el viejo» ......................... $\quad 380$

De Gómez «el moço» $\ldots \ldots \ldots \ldots \ldots \ldots \ldots \ldots \ldots \ldots \ldots \ldots \ldots \ldots . . . \ldots \ldots$

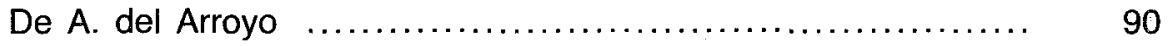

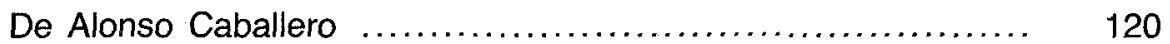

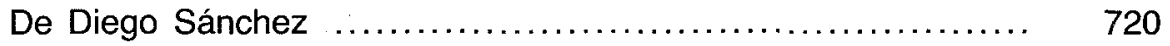

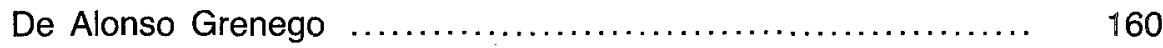

De Pedro López (herrero) $\ldots \ldots \ldots \ldots \ldots \ldots \ldots \ldots \ldots \ldots \ldots \ldots . . . \ldots \ldots$

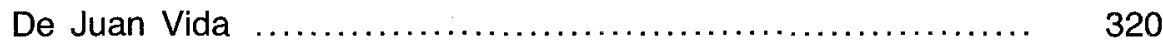

De Francisco Sánchez Corvacho $\ldots \ldots \ldots \ldots \ldots \ldots \ldots \ldots \ldots \ldots \ldots \ldots \ldots$

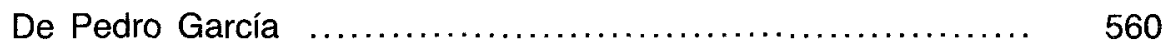

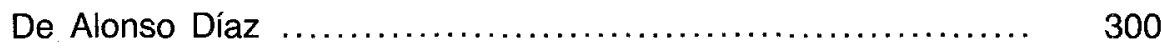


Mrs.

De Miguel Díaz 180

De Alonso del Arroyo 180

De Sebastián Grande

De la mujer de Andrés de Segovia

De Gabriel de Requena

De Juan de Viñas «el moço»

De Francisco Marin

De Alonso Romero

De Juan Vida y Francisco de Hernán García, de Luis Cano y de Andrés Gómez, de Juan Díaz de Mora, de Francisco Moreno, de Juan López, de B. Sánchez Corbacho, de F. de Relonga y de Blas de Vega

De Andrés de Segovia

De Juan García

De Juan Sánchez Laso

De Miguel Sánchez, hijo de Andrés de Segovia

De Antonia... viuda

De B. Moreno (herrero)

De Miguel Sánchez de Villarrubia 
Mrs.

De Antón Ramírez «el moço»

De Martín Ramírez (sastre)

De Antón Garcia

De Juan Fernández

De A. Lumbreras

De Luis

De Diego Marín

De Juan Sánchez (talabartero)

De Martín Gallego 560

De Juan Sánchez de Tembleque 700

De Andrés Garcia 140

De Francisco Hernández de La Guardia 40

De Francisco de Ayllón

De Diego Martín

De Alonso Serrano

De Andrés

De Martín González

De Benito García

De Pedro Grueso 
Mrs.

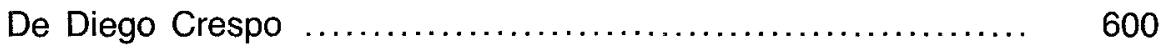

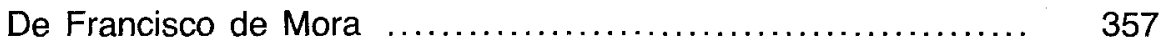

De Juan de Revenga $\ldots \ldots \ldots \ldots \ldots \ldots \ldots \ldots \ldots \ldots \ldots \ldots \ldots . \ldots \ldots \ldots$

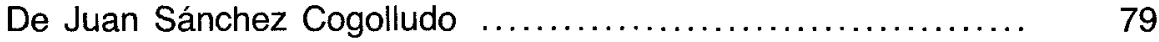

De Cristóbal García $\ldots \ldots \ldots \ldots \ldots \ldots \ldots \ldots \ldots \ldots \ldots \ldots \ldots \ldots \ldots, \quad 453$

De A. López de la Fuente ................................. 148

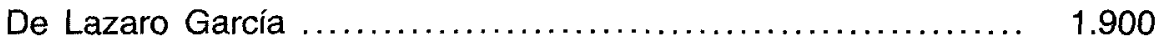

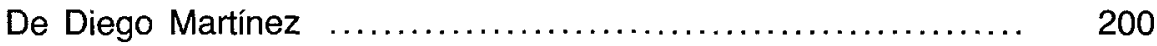

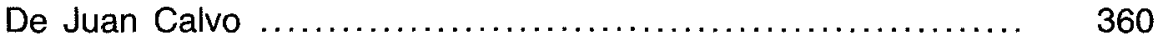

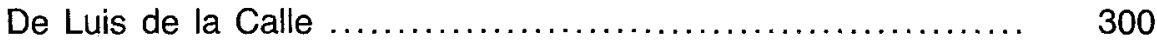

TOTAL $\ldots \ldots \ldots \ldots \ldots \ldots \ldots \ldots \ldots \ldots \ldots \ldots \ldots \ldots \ldots \ldots \ldots \ldots \ldots \ldots \ldots \ldots \ldots \ldots \ldots \ldots, 32.471$ 


\section{APENDICE ॥}

RELACION DE LAS VENTAS EFECTUADAS DE VIÑAS

\begin{tabular}{|c|c|c|c|c|c|}
\hline & FECHA & VENDEDOR & COMPRADOR & CANTIDAD & PRECIO (MRS) \\
\hline \multirow[t]{4}{*}{1564} & (24 enero) & Alonso Rei «El moço" & $\begin{array}{l}\text { Pedro Pérez } \\
\text { (sastre) }\end{array}$ & 3,5 aranzadas & 3.353 \\
\hline & & $\begin{array}{l}\text { Juan Pérez (guarda de } \\
\text { Yegros) }\end{array}$ & Diego García & $\begin{array}{l}1 \text { majuelo } \\
1 / 2 \text { molino }\end{array}$ & $\begin{array}{l}16.300 \\
10.000\end{array}$ \\
\hline & & $\begin{array}{l}\text { Cristóbal García } \\
\text { (mesoriero) }\end{array}$ & $\begin{array}{l}\text { Francisco de } \\
\text { los Santos }\end{array}$ & $\begin{array}{l}1 \text { majuelo de } \\
2.000 \text { sar- } \\
\text { mientos }\end{array}$ & 17.000 \\
\hline & & Alonso López & $\begin{array}{l}\text { Francisco Gómez } \\
\text { de Aparicio }\end{array}$ & $\begin{array}{l}1 \text { majuelo de } \\
2.200 \text { sar- } \\
\text { mientos }\end{array}$ & 14.300 \\
\hline \multirow[t]{7}{*}{1565} & (9 julio) & Pedro Gómez & Cristóbal López & $\begin{array}{l}1 \text { majuelo de } \\
2.400 \text { plantas }\end{array}$ & 18.000 \\
\hline & (29 julio) & Gabriel S. & Antón Ramírez & 1 majuelo & 23.625 \\
\hline & $(23 \mathrm{ag})$. & Pedro Serrano & Aintón Ramírez & 1 majuelo & 14.439 \\
\hline & (28 ag.) & Benito Fernández & Juan Sánchez & $\begin{array}{l}1 \text { majuelo de } \\
1.000 \text { plantas }\end{array}$ & 24.000 \\
\hline & (21 sept.) & Alonso del Arroyo & Juan de S. & $\begin{array}{l}1 \text { majuelo y } 1 \\
\text { tierra }\end{array}$ & 4.500 \\
\hline & (30 dic.) & Miguel Díaz & Pedro Gómez & 1 majuelo & 9.000 \\
\hline & (30 dic.) & Alonso Díaz & Pedro Gómez & 1 majuelo & 15.000 \\
\hline \multirow[t]{2}{*}{1566} & (24 mayo) & Andrés de Segovia & M. Ramírez & 393 cepas & 3.820 \\
\hline & (31 julio) & Gabriel de Requena & Juan Portes & 1 majuelo & 12.000 \\
\hline
\end{tabular}


PAULINA LÓPEZ PITA

\begin{tabular}{|c|c|c|c|c|c|}
\hline & FECHA & VENDEDOR & COMPRADOR & CANTIDAD & PRECIO (MRS) \\
\hline & (28 ag.) & Luis de la Calle & Juan López & 1 majuelo & 15.200 \\
\hline & (6 sept.) & Diego Crespo & Francisco Rosado & 1 majuelo & 30.000 \\
\hline & (24 oct.) & G. de Sesa & Antonio Ramírez & 1 majuelo & 24.000 \\
\hline & (24 oct.) & Pedro Serrano & Antonio Ramírez & 1 majuelo & 12.500 \\
\hline & (1 nov.) & Alonso de Lillo & Alonso Sánchez & 1 majuelo & 4.125 \\
\hline & (6 nov.) & Juan Sánchez & Antonio García & $\begin{array}{l}1 \text { majuelo de } \\
3.017 \text { plantas }\end{array}$ & 39.000 \\
\hline & (13 nov.) & Juan Díaz & Alonso de Escalona & 1 majuelo & 4.150 \\
\hline & (18 nov.) & Diego Marín & Juan de $R$. & $\begin{array}{l}1 \text { majuelo de } \\
1.000 \text { cepas }\end{array}$ & 12.238 \\
\hline & $(20$ nov. $)$ & Antón García & Juan Sánchez Laso & $\begin{array}{l}1 \text { majuelo de } \\
1.480 \text { cepas }\end{array}$ & 24.820 \\
\hline & (8 dic.) & Pedro Grueso & Alonso Maestro & 1 majuelo & 4.500 \\
\hline & (15 dic.) & Luisa López & Aparicio Sánchez & $\begin{array}{l}1 \text { majuelo de } \\
2.000 \text { cepas }\end{array}$ & 17.000 \\
\hline & (20 dic.) & Juan Fernández & Juan Gómez & $\begin{array}{l}1 \text { majuelo de } \\
731 \text { cepas }\end{array}$ & 10.965 \\
\hline & (23 dic.) & Alonso Grueso & $\begin{array}{l}\text { Alonso Fernández } \\
\text { del Cañaveral }\end{array}$ & $\begin{array}{l}1 \text { majuelo de } \\
1.500 \text { plantas }\end{array}$ & 27.000 \\
\hline 1567 & (3 enero) & Bartolomé Romero & Diego García & $\begin{array}{l}1 \text { majuelo de } \\
1.600 \text { cepas }\end{array}$ & 27.010 \\
\hline & (19 enero) & Gabriel Minguez & Cristóbal G. & $\begin{array}{l}1 \text { majuelo de } \\
1.600 \text { cepas }\end{array}$ & 14.000 \\
\hline & (19 enero) & Cristóbal García & Alonso Díaz & 1 majuelo & 22.500 \\
\hline & (19 enero) & $\begin{array}{l}\text { Alonso López de } \\
\text { Fuente }\end{array}$ & Bariolomé Díaz & 1 majuelo & 7.400 \\
\hline & (20 enero) & $\begin{array}{l}\text { Miguel López de On- } \\
\text { rubia }\end{array}$ & Martín Díaz & $\begin{array}{l}1 \text { majuelo de } \\
1.014 \text { cepas }\end{array}$ & 21.760 \\
\hline & (24 enero) & Luis Ramos & Quiteria Gómez & 1 majuelo & 23.000 \\
\hline
\end{tabular}


Un nuevo dato sobre la enajenación de bienes de la Corona

\begin{tabular}{|c|c|c|c|c|}
\hline FECHA & VENDEDOR & COMPRADOR & CANTIDAD & PRECIO (MRS) \\
\hline$(2 \mathrm{feb})$. & Juan García & Juan Gómez & $\begin{array}{l}1 \text { majuelo de } \\
1.440 \text { cepas }\end{array}$ & 18.000 \\
\hline (3 feb.) & Juan Calvo & - & 1 majuelo & 8.160 \\
\hline (14 feb.) & $\begin{array}{l}\text { Martín Gallego } \\
\text { (sastre) }\end{array}$ & $\begin{array}{l}\text { Juan Sánchez } \\
\text { (Talabartero) }\end{array}$ & 1 majuelo & 28.000 \\
\hline (15 feb.) & María de Toledo & Alonso Maestro & $\begin{array}{l}4 \text { pedazos de } \\
\text { tierra }\end{array}$ & 30.300 \\
\hline (4 marzo) & Juan Sánchez Vegue & María Pérez & $\begin{array}{l}1 \text { majuelo de } \\
1.000 \text { cepas }\end{array}$ & 21.319 \\
\hline (2 abril) & Diego López & Antonio Ramírez & 1 majuelo & 20.000 \\
\hline (1 julio) & Antón García & Pedro López & $\begin{array}{l}1 \text { majuelo de } \\
1.313 \text { cepas }\end{array}$ & 17.000 \\
\hline (25 ag.) & Luis Martín & $\begin{array}{l}\text { Juan Hernández de } \\
\text { las Higueras }\end{array}$ & $\begin{array}{l}1 \text { majuelo de } \\
1.400 \text { cepas }\end{array}$ & 15.000 \\
\hline (29 sept.) & Martín Díaz & B. de Ortega & $\begin{array}{l}1 \text { majuelo de } \\
2.000 \text { cepas }\end{array}$ & 39.340 \\
\hline (19 oct.) & Fernán González & T. de Ortega & 1 majuelo & 14.592 \\
\hline (19 oct.) & Benito García & $\begin{array}{l}\text { Luis Hernández Pin- } \\
\text { tado }\end{array}$ & 1 majuelo & 8.625 \\
\hline (28 oct.) & Blas de Vega & Cristóbal & 1 majuelo & 442.000 \\
\hline$(20$ nov. $)$ & $\begin{array}{l}\text { Cristóbal Sánchez } \\
\text { (tejero) }\end{array}$ & Gonzalo López & 1 majuelo & 21.435 \\
\hline$(3$ dic.) & Diego García & $\begin{array}{l}\text { Juan Martín de la } \\
\text { Rosa }\end{array}$ & 1 majuelo & 6.000 \\
\hline (10 dic.) & Martín Ramírez & Juan García Hortelano & $\begin{array}{l}1 \text { majuelo de } \\
1.618 \text { cepas }\end{array}$ & 37.214 \\
\hline (17 dic.) & M. González & - & 1 majuelo & 10.000 \\
\hline (30 dic.) & Blas Maestro & Andrés Gómez & $\begin{array}{l}1 \text { majuelo de } \\
1.440 \text { cepas }\end{array}$ & 24.480 \\
\hline
\end{tabular}


